COMMUNICATIONS IN

ANALYSIS AND GEOMETRY

Volume 11, Number 2, 235-261, 2003

\title{
Interior Curvature Bounds For Spacelike Hypersurfaces Of Prescribed $k$-th Mean Curvature
}

\author{
JOHN URBAS
}

\begin{abstract}
We derive interior curvature bounds for strictly spacelike hypersurfaces of prescribed $k$-th mean curvature in Minkowski space analogous to those we derived in previous work on the Euclidean case. The estimates depend on a sufficiently large $L^{p}$ norm of the mean curvature. Examples similar to those in the Euclidean case show that if $k \geq 3$, such curvature bounds are generally false if $p$ is not sufficiently large.
\end{abstract}

\section{Introduction.}

In this paper we derive interior curvature bounds for strictly spacelike $k$ admissible hypersurfaces of prescribed $k$-th mean curvature in Minkowski space $\mathbf{L}^{n+1}$ analogous to those we derived in $[19,20]$ in the Euclidean case. The results and techniques are similar in many respects. However, in the Minkowski case certain key curvature terms have the opposite sign to that encountered in the Euclidean case. This leads to significant differences in the proofs. For this reason we believe it is worthwhile providing a largely self-contained proof of the estimates in the Minkowski case.

Up to now little attention has been given to hypersurfaces of prescribed $k$-th mean curvature in Minkowski space and in more Lorentzian manifolds if $k \geq 2$. There are many papers on maximal hypersurfaces, and more generally, on hypersurfaces of prescribed mean curvature (see $[2,3,6,8,10,11]$ ). The Gauss curvature case $k=n$ has been studied by Delanoë [7] and Guan [13]. Very recently Bayard $[4,5]$ established the classical solvability of the Dirichlet problem in the scalar curvature case $k=2$ with $n=3,4$. We mention also the recent work of Gerhardt [12] and Schnürer [17] on curvature

\footnotetext{
${ }^{1}$ This work was begun at the Isaac Newton Institute for Mathematical Sciences at Cambridge University during March and April 2001. The author thanks the institute for its support during this time. The author was also supported by an Australian Research Council Senior Fellowship.
} 
equations in Lorentzian manifolds. In these works the equations are modelled on the Gauss curvature; the $k$-th mean curvature with $k<n$ is excluded.

Minkowski space $\mathbf{L}^{n+1}$ is the space $\mathbf{R}^{n} \times \mathbf{R}$ equipped with the metric

$$
d s^{2}=d x_{1}^{2}+\cdots+d x_{n}^{2}-d x_{n+1}^{2} .
$$

We are interested in hypersurfaces $M$ which are graphs of a strictly spacelike function $u$ defined over a subdomain of $\mathbf{R}^{n}$. Since we are concerned here only with local estimates, we assume that $u \in C^{4}\left(\bar{B}_{d_{0}}\right)$ is strictly spacelike, meaning that

$$
\sup _{B_{d_{0}}}|D u| \leq \theta
$$

for a positive constant $\theta<1$. Here $B_{d_{0}}$ denotes the open ball in $\mathbf{R}^{n}$ of radius $d_{0}$ centred at the origin.

If $M=\operatorname{graph} u$, the Minkowski metric (1.1) restricted to $M$ defines a Riemannian metric on $M$, which in the standard coordinates on $\mathbf{L}^{n+1}$ is given by

$$
g_{i j}=\delta_{i j}-D_{i} u D_{j} u, \quad 1 \leq i, j \leq n .
$$

The inverse of the metric is

$$
g^{i j}=\delta_{i j}+\frac{D_{i} u D_{j} u}{1-|D u|^{2}}
$$

The second fundamental form of $M$ is given by

$$
h_{i j}=\frac{D_{i j} u}{\sqrt{1-|D u|^{2}}}
$$

The principal curvatures $\lambda_{1}, \ldots, \lambda_{n}$ of $M$ are the eigenvalues of $\left[h_{i j}\right]$ relative to $\left[g_{i j}\right]$.

The $k$-th mean curvature $H_{k}$ of $M$ is defined to be the $k$-th elementary symmetric function of the principal curvatures,

$$
H_{k}=S_{k}\left(\lambda_{1}, \ldots, \lambda_{n}\right)=\sum_{1 \leq i_{1}<\cdots<i_{k} \leq n} \lambda_{i_{1}} \cdots \lambda_{i_{k}}
$$

As in the Euclidean case we say that $M$ (or $u$ ) is $k$-admissible if at each point its vector of principal curvatures $\lambda=\left(\lambda_{1}, \ldots, \lambda_{n}\right)$ belongs to the cone

$$
\Gamma_{k}=\left\{\lambda \in \mathbf{R}^{n}: S_{j}(\lambda)>0, j=1, \ldots, k\right\} .
$$

In the Euclidean case it is well known that the $k$-th mean curvature equation

$$
H_{k}[u]=\psi
$$


is an elliptic equation and $H_{k}[u]^{1 / k}$ is a concave function of $D^{2} u$ if $u$ is $k$ admissible. The same is of course true for strictly spacelike, $k$-admissible solutions in the Minkowski case.

The main result of this paper is the following interior curvature bound.

Theorem 1.1. Let $n \geq 3$ and $k \in\{2, \ldots, n\}$. Let $u \in C^{4}\left(\bar{B}_{d_{0}}\right)$ be a strictly spacelike $k$-admissible solution of

$$
H_{k}[u]^{1 / k}=g \quad \text { in } \quad B_{d_{0}}
$$

where $g$ satisfies

$$
\mu^{-1} \leq g \leq \mu, \quad|\nabla g| \leq \mu \quad \text { on } \quad M
$$

for some positive constant $\mu$. Then for any $s>k(n-1) / 2$ we have

$$
|A(0)| \leq C
$$

where $A$ is the second fundamental form of $M$ and $C$ depends only on $k, n, s, \mu, d_{0}$, the constant $\theta$ in $(1.2)$, and on $\int_{M} H_{1}^{s}$.

Remarks. (i) This parallels the interior curvature bound of [20], which is essentially the same result with (1.2) replaced by control of the modulus of continuity of $\nu$, which is a stronger assumption. In effect, the result in the Euclidean case holds for graphs with sufficiently small gradient. Such a restriction is not explicitly assumed in the Minkowski case - it is not needed if $k=2$, and if $k \geq 3$, it is implied by the integrability hypothesis on $H_{1}$ and the Sobolev embedding theorem.

(ii) The same arguments as in the Euclidean case can be used to show that the estimate (1.11) is false if $k \geq 3$ and if $s$ is not sufficiently large. Namely, well known examples of Pogorelov and of Caffarelli (see [19]) can be used as comparison functions to show that for $k \geq 3$ and for sufficiently small positive $\lambda$ and $\epsilon$ the equation of prescribed Lorentz-Gauss curvature

$$
\operatorname{det} D^{2} u=\lambda\left(1-|D u|^{2}\right)^{(k+2) / 2} \quad \text { in } \quad B_{\epsilon}^{k} \subset \mathbf{R}^{k}
$$

has convex, strictly spacelike generalized (viscosity) solutions which are Lipschitz continuous but not $C^{1}$ in the interior, or which belong to $C^{1,1-2 / k} \cap W^{2, p}$ for all $p<k(k-1) / 2$. By extending such solutions to be constant in the $x_{k+1}, \ldots, x_{n}$ coordinate directions we obtain viscosity solutions of (1.8) with $\psi$ a positive constant and having the same regularity. This shows that for $k \geq 3$ purely interior curvature bounds for strictly spacelike $k$-admissible 
solutions of (1.8) do not hold in general, and moreover, even some control of the solution in $C^{1, \alpha}$ or in $W^{2, p}$ may not be sufficient to imply higher bounds.

(iii) In the case $k=n$ the lower bound on $s$ is known to be essentially sharp (see [18]). We do not know whether the lower bound $s>k(n-1) / 2$ is optimal if $k<n$.

\section{A preliminary curvature bound.}

The curvature bound will be proved in two main steps, as in the Euclidean case. First we will prove an interior curvature bound depending on $\int_{M} H_{1}^{p}$ for some $p>k n / 2$, and second, for some $p>k n / 2$ and any $M^{\prime} \subset \subset M$ we will derive a bound for $\int_{M^{\prime}} H_{1}^{p}$, depending on $\int_{M} H_{1}^{s}$ for some $s>k(n-1) / 2$. In this section we carry out the first step.

We begin by recalling the structure conditions satisfied by the $k$-th mean curvature equation. We will work in a local orthonormal frame field $\hat{\mathbf{e}}_{1}, \ldots, \hat{\mathbf{e}}_{n}$ defined on $M$ near $(0, u(0))$. Covariant differentiation on $M$ in the direction $\hat{\mathbf{e}}_{i}$ will be denoted by $\nabla_{i}$. We denote the components of the second fundamental form $A$ in this frame by $h_{i j}$. Thus $h_{i j}=\left\langle D_{\hat{\mathbf{e}}_{i}} \nu, \hat{\mathbf{e}}_{j}\right\rangle$ where $D$ is the connection on $\mathbf{L}^{n+1},\langle\cdot, \cdot\rangle$ is the Minkowski inner product, and $\nu$ is the upward (future directed) unit normal

$$
\nu=\frac{(D u, 1)}{\sqrt{1-|D u|^{2}}}
$$

With these sign conventions the second fundamental form of the graph of a convex strictly spacelike function is nonnegative.

Equation (1.9) can be written as

$$
H_{k}[A]=g^{k} .
$$

As in the Euclidean case, $\left(H_{k}[u]\right)^{1 / k}$ is a concave function of $\left[h_{i j}\right]$ if $M$ is strictly spacelike and $k$-admissible. Furthermore, the ellipticity bounds are

$$
\frac{g^{k}}{H_{1}} I \leq\left[F_{i j}\right] \leq(n-k+1) H_{k-1} I
$$

where

$$
F_{i j}=\frac{\partial H_{k}}{\partial a_{i j}}[A]
$$

The other key fact we will use is that $F_{i j}$ is divergence free:

$$
\nabla_{i} F_{i j}=0
$$


All these facts can be proved in the same way as in the Euclidean case. To prove (2.4) we use the Codazzi equations, which tell us that $\nabla_{k} h_{i j}$ is symmetric in all indices.

We need to compute a differential inequality for the mean curvature $H_{1}$. To do this we write $(2.2)$ in the form

$$
G_{k}[A]:=H_{k}[A]^{1 / k}=g .
$$

Differentiating (2.5) and writing $G_{i j}=\frac{\partial G_{k}}{\partial a_{i j}}[A]$, we obtain

$$
\begin{aligned}
& G_{i j} \nabla_{l} h_{i j}=\nabla_{l} g, \\
& G_{i j} \nabla_{l} \nabla_{l} h_{i j} \geq \nabla_{l} \nabla_{l} g=\Delta g,
\end{aligned}
$$

where in the last inequality we have used the concavity of $G$ to discard a term which is quadratic in $\nabla A$, and where $\Delta$ denotes the Laplace-Beltrami operator on $M$. As usual we assume summation from 1 to $n$ over repeated indices. Using the Codazzi equations, the standard formula for commuting covariant derivatives, and the Gauss equations, which in Minkowski space are

$$
R_{i j k l}=-\left(h_{i k} h_{j l}-h_{i l} h_{j k}\right)
$$

with $R_{i j k l}$ denoting the Riemann curvature tensor, we find that

$$
\begin{aligned}
\nabla_{l} \nabla_{l} h_{i j}= & \nabla_{l} \nabla_{i} h_{j l} \\
= & \nabla_{i} \nabla_{l} h_{j l}+R_{l i j m} h_{m l}+R_{l i l m} h_{m j} \\
= & \nabla_{i} \nabla_{j} h_{l l}-h_{l j} h_{i m} h_{m l}+h_{l m} h_{i j} h_{m l} \\
& -h_{l l} h_{i m} h_{m j}+h_{l m} h_{i l} h_{m j} \\
= & \nabla_{i} \nabla_{j} h_{l l}+h_{l m} h_{i j} h_{m l}-h_{l l} h_{i m} h_{m j} .
\end{aligned}
$$

Using this in (2.6) we obtain

$$
G_{i j} \nabla_{i} \nabla_{j} h_{l l} \geq G_{i j} h_{i m} h_{j m} h_{l l}-G_{i j} h_{i j} h_{l m} h_{l m}+\Delta g
$$

We have

$$
G_{i j}=\frac{1}{k} H_{k}^{(1-k) / k} F_{i j}=\frac{1}{k} g^{1-k} F_{i j}
$$

so using this in (2.7) we obtain

$$
F_{i j} \nabla_{i} \nabla_{j} H_{1}=F_{i j} \nabla_{i} \nabla_{j} h_{l l} \geq H_{1} F_{i j} h_{i m} h_{j m}-F_{i j} h_{i j} h_{l m} h_{l m}+k g^{k-1} \Delta g .
$$

Observe that if $\Delta g \geq 0$, the last term can be dropped; the subsequent computations involving this term are then unnecessary, and some of the 
intermediate estimates, which are of independent interest, have a simpler form.

The first two terms on the right hand side of (2.9) have the opposite sign to that encountered in the Euclidean case. In the Minkowski case it is $F_{i j} h_{i j} h_{l m} h_{l m}$ that needs to be controlled, while in the Euclidean case it is $H_{1} F_{i j} h_{i m} h_{j m}$. This leads to significant differences in the proof.

We will use certain cutoff functions in the proof. These will be defined using the Lorentz distance from the origin restricted to $M$ (we assume for convenience that $u(0)=0$ ), which is defined by

$$
l(X)=\sqrt{|x|^{2}-|u(x)|^{2}}, \quad X=(x, u(x)) .
$$

The open Lorentz ball $L_{\rho}$ of radius $\rho$ centred at the origin is

$$
L_{\rho}=\left\{\left(y, y_{n+1}\right) \in \mathbf{L}^{n+1}: \sqrt{|y|^{2}-y_{n+1}^{2}}<\rho\right\} .
$$

We let $M_{\rho}=M \cap L_{\rho}$. Then a simple computation shows that

$$
M_{\rho} \subset B_{\rho / \sqrt{1-\theta^{2}}} \times \mathbf{R},
$$

where $B_{r}$ denotes the open ball of radius $r$ in $\mathbf{R}^{n}$ centred at the origin. Furthermore, $\partial M_{\rho}=M \cap \partial L_{\rho}$ if $\partial M \cap L_{\rho}=\emptyset$, because $M$ is strictly spacelike.

By direct computation we have

$$
|\nabla l|^{2}=1+(l(X))^{-2}\langle X, \nu\rangle^{2}=1+(l(X))^{-2}\left(\frac{x \cdot D u-u}{\sqrt{1-|D u|^{2}}}\right)^{2} .
$$

Since $u(0)=0$ and $u$ satisfies (1.2), we have $|u(x)| \leq \theta|x|$, and therefore

$$
1 \leq|\nabla l|^{2} \leq 1+\frac{4}{\left(1-\theta^{2}\right)^{2}}
$$

We now let

$$
R=\sqrt{1-\theta^{2}} d_{0}
$$

so that $\partial M \cap L_{R}=\emptyset$. For any $\rho \in(0, R / 2]$ we choose a nonnegative function $\zeta \in C_{0}^{2}([0,2 \rho))$ such that $0 \leq \zeta(t) \leq 1, \zeta(t)=1$ for $0 \leq t \leq \rho$, and $\left|\zeta^{\prime}\right| \leq 2 \rho^{-1}$. We let $\eta(X)=\zeta(l(X))$. Then, from (2.14) we have

$$
|\nabla \eta| \leq \frac{C(\theta)}{\rho}
$$


In various integrals over $n$-dimensional subsets of $M$ we will usually omit the measure, because most integrals will be with respect to the measure $\mu_{L}$ induced by the Minkowski metric. Where necessary we will indicate this to distinguish integrals with respect to the measure $\mu_{E}$ induced on $M$ by regarding $M$ as a subset of $\mathbf{R}^{n} \times \mathbf{R}$ with the usual Euclidean structure. At certain points in Sections 3 and 4 we will need to consider integrals over $n-1$ dimensional subsets of $M$. The $n-1$ dimensional measures will be denoted by $\sigma_{L}$ and $\sigma_{E}$ according to whether $M$ is regarded as a subset of $\mathbf{R}^{n} \times \mathbf{R}$ with the Minkowski or Euclidean metric respectively.

We now multiply $(2.9)$ by $\eta^{2} H_{1}^{p}$ for some $p>0$ to be specified later. Integrating by parts twice and using (2.4), we obtain

$$
\begin{aligned}
& \int_{M_{2 \rho}} \eta^{2} H_{1}^{p}\left(H_{1} F_{i j} h_{i m} h_{j m}-F_{i j} h_{i j} h_{l m} h_{l m}+k g^{k-1} \Delta g\right) \\
& \leq \int_{M_{2 \rho}} \eta^{2} H_{1}^{p} F_{i j} \nabla_{i} \nabla_{j} H_{1} \\
& =-2 \int_{M_{2 \rho}} \eta H_{1}^{p} F_{i j} \nabla_{i} \eta \nabla_{j} H_{1}-p \int_{M_{2 \rho}} \eta^{2} H_{1}^{p-1} F_{i j} \nabla_{i} H_{1} \nabla_{j} H_{1} \\
& \leq \frac{C}{p} \int_{M_{2 \rho}} H_{1}^{p+1} F_{i j} \nabla_{i} \eta \nabla_{j} \eta-\frac{3 p}{4} \int_{M_{2 \rho}} \eta^{2} H_{1}^{p-1} F_{i j} \nabla_{i} H_{1} \nabla_{j} H_{1} \\
& \leq \frac{C}{p \rho^{2}} \int_{M_{2 \rho}} H_{1}^{p+1} H_{k-1}-\frac{3 p}{4} \int_{M_{2 \rho}} \eta^{2} H_{1}^{p-1} F_{i j} \nabla_{i} H_{1} \nabla_{j} H_{1}
\end{aligned}
$$

where to obtain the last line we have used (2.15) and

$$
\sum_{i=1}^{n} F_{i i}=(n-k+1) H_{k-1}
$$

Since $\eta=0$ on $\partial M_{2 \rho}$,

$$
\begin{aligned}
-\int_{M_{2 \rho}} \eta^{2} H_{1}^{p} g^{k-1} \nabla_{l} \nabla_{l} g= & \int_{M_{2} \rho} \nabla_{l}\left[\eta^{2} g^{k-1} H_{1}^{p}\right] \nabla_{l} g \\
= & 2 \int_{M_{2 \rho}} \eta g^{k-1} H_{1}^{p} \nabla_{l} g \nabla_{l} \eta \\
& +(k-1) \int_{M_{2 \rho}} \eta^{2} g^{k-2} H_{1}^{p}|\nabla g|^{2} \\
& +p \int_{M_{2 \rho}} \eta^{2} g^{k-1} H_{1}^{p-1} \nabla_{l} H_{1} \nabla_{l} g .
\end{aligned}
$$

To control the first term we estimate

$$
\begin{aligned}
& \left|\int_{M_{2 \rho}} \eta g^{k-1} H_{1}^{p} \nabla_{l} g \nabla_{l} \eta\right| \\
& \leq \frac{1}{p} \int_{M_{2 \rho}} H_{1}^{p+1} F_{i j} \nabla_{i} \eta \nabla_{j} \eta+p \int_{M_{2 \rho}} \eta^{2} g^{2 k-2} H_{1}^{p-1} F_{i j}^{-1} \nabla_{i} g \nabla_{j} g \\
& \leq \frac{C}{p \rho^{2}} \int_{M_{2 \rho}} H_{1}^{p+1} H_{k-1}+p \int_{M_{2 \rho}} g^{k-2}|\nabla g|^{2} H_{1}^{p},
\end{aligned}
$$

where to obtain the last integral we have used the inequality

$$
\left[F_{i j}\right] \geq \frac{g^{k}}{H_{1}} I
$$


or more precisely, its equivalent form

$$
\left[F_{i j}^{-1}\right] \leq \frac{H_{1}}{g^{k}} I .
$$

These inequalities are proved in [19].

The third term on the right hand side of (2.18) can be estimated similarly. We obtain

$$
\begin{aligned}
& \left|p \int_{M_{2 \rho}} \eta^{2} g^{k-1} H_{1}^{p-1} \nabla_{l} H_{1} \nabla_{l} g\right| \\
& \leq \frac{p}{4} \int_{M_{2 \rho}} \eta^{2} H_{1}^{p-1} F_{i j} \nabla_{i} H_{1} \nabla_{j} H_{1}+C p \int_{M_{2 \rho}} \eta^{2} g^{2 k-2} H_{1}^{p-1} F_{i j}^{-1} \nabla_{i} g \nabla_{j} g \\
& \leq \frac{p}{4} \int_{M_{2 \rho}} \eta^{2} H_{1}^{p-1} F_{i j} \nabla_{i} H_{1} \nabla_{j} H_{1}+C p \int_{M_{2 \rho}} g^{k-2}|\nabla g|^{2} H_{1}^{p} .
\end{aligned}
$$

Combining these estimates with (2.16) and using the facts that

$$
F_{i j} h_{i j}=k g^{k}
$$

and

$$
h_{l m} h_{l m}=|A|^{2} \leq H_{1}^{2},
$$

which follows since $M$ is 2 -admissible, we obtain

$$
\begin{aligned}
& \frac{p}{2} \int_{M_{2 \rho}} \eta^{2} H_{1}^{p-1} F_{i j} \nabla_{i} H_{1} \nabla_{j} H_{1} \leq k \int_{M_{2 \rho}} \eta^{2} g^{k} H_{1}^{p+2} \\
& \quad+C_{1}(1+p) \int_{M_{2 \rho}} g^{k-2}|\nabla g|^{2} H_{1}^{p}+\frac{C_{2}}{p \rho^{2}} \int_{M_{2 \rho}} H_{1}^{p+1} H_{k-1},
\end{aligned}
$$

where $C_{1}, C_{2}$ are positive constants depending only on $k$ and $n$. Simplifying this using the bounds (1.10) for $g$ and $|\nabla g|$ we obtain

$$
\begin{aligned}
& p \int_{M_{2 \rho}} \eta^{2} H_{1}^{p-1} F_{i j} \nabla_{i} H_{1} \nabla_{j} H_{1} \\
& \leq C\left\{\int_{M_{2 \rho}} \eta^{2} H_{1}^{p+2}+(1+p) \int_{M_{2 \rho}} H_{1}^{p}+\frac{1}{p \rho^{2}} \int_{M_{2 \rho}} H_{1}^{p+1} H_{k-1},\right\}
\end{aligned}
$$

where $C$ depends only on $k, n$ and $\mu$. We now estimate the integral on the left from below using the lower ellipticity bound (2.20):

$$
\begin{aligned}
p \int_{M_{2 \rho}} \eta^{2} H_{1}^{p-1} F_{i j} \nabla_{i} H_{1} \nabla_{j} H_{1} & \geq \frac{4}{p} \int_{M_{2 \rho}} \eta^{2} g^{k}\left|\nabla\left(H_{1}^{p / 2}\right)\right|^{2} \\
& \geq \frac{2}{p \mu^{k}} \int_{M_{2 \rho}}\left|\nabla\left(\eta H_{1}^{p / 2}\right)\right|^{2}-\frac{C}{p \rho^{2}} \int_{M_{2 \rho}} H_{1}^{p},
\end{aligned}
$$

where we have once again used (2.15). Using this in (2.25) we obtain

$$
\begin{aligned}
& \int_{M_{2 \rho}}\left|\nabla\left(\eta H_{1}^{p / 2}\right)\right|^{2} \\
& \leq C\left\{p \int_{M_{2 \rho}} \eta^{2} H_{1}^{p+2}+\frac{1+p^{2}}{\rho^{2}} \int_{M_{2 \rho}} H_{1}^{p}+\frac{1}{\rho^{2}} \int_{M_{2 \rho}} H_{1}^{p+1} H_{k-1},\right\} .
\end{aligned}
$$


We now want to apply a Sobolev inequality. We will use the Sobolev inequality of Allard [1] and Michael and Simon [14], which is valid for submanifolds $M$ of Euclidean space. In our case $M$ is not a submanifold of Euclidean space, but we may use the Euclidean version and then estimate various Euclidean quantities by the corresponding Minkowski quantities. To be more precise, we indicate the metric, second fundamental form, connection and measure on $M$ induced by the Euclidean metric on $\mathbf{R}^{n} \times \mathbf{R}$ by a subscript or superscript $E$, whichever is more convenient. Where necessary we use a subscript or superscript $L$ to indicate quantities in the Minkowski metric. Thus

$$
\begin{aligned}
g_{i j}^{E} & =\delta_{i j}+D_{i} u D_{j} u, \\
g_{E}^{i j} & =\delta_{i j}-\frac{D_{i} u D_{j} u}{1+|D u|^{2}}, \\
h_{i j}^{E} & =\frac{D_{i j} u}{\sqrt{1+|D u|^{2}}} .
\end{aligned}
$$

The Sobolev inequality of Allard, Michael and Simon states that for any function $w \in C_{0}^{1}(M)$ and any $r \in[1, n)$ we have

$$
\begin{aligned}
& \left(\int_{M}|w|^{n r /(n-r)} d \mu_{E}\right)^{(n-r) / n r} \\
& \leq C(n, r)\left(\int_{M}\left|\nabla^{E} w\right|^{r} d \mu_{E}+\int_{M}\left|H_{M}^{E}\right|^{r}|w|^{r} d \mu_{E}\right)^{1 / r}
\end{aligned}
$$

where $H_{M}^{E}$ denotes the Euclidean mean curvature of $M$,

$$
H_{M}^{E}=g_{E}^{i j} h_{i j}^{E}=\frac{1}{\sqrt{1+|D u|^{2}}}\left(\delta_{i j}-\frac{D_{i} u D_{j} u}{1+|D u|^{2}}\right) D_{i j} u .
$$

Since $M$ is strictly spacelike, the Euclidean and Minkowski quantities are comparable. This is straightforward to verify; we do this only for the mean curvatures. We have

$$
\begin{aligned}
H_{M}^{E} & =g_{E}^{i j} h_{i j}^{E} \\
& =g_{L}^{i j} h_{i j}^{L}+\left(\frac{g_{E}^{i j}}{\sqrt{1+|D u|^{2}}}-\frac{g_{L}^{i j}}{\sqrt{1-|D u|^{2}}}\right) D_{i j} u \\
& \leq H_{1}+C_{1}(n, \theta)\left|D^{2} u\right| \\
& \leq C_{2}(n, \theta) H_{1},
\end{aligned}
$$

where $\theta$ is the constant from (1.2). Since also $\left|\nabla^{E} w\right| \leq\left|\nabla^{L} w\right|$, we see that the Sobolev inequality (2.27) is valid if all Euclidean quantities are replaced by the corresponding Minkowski quantities; the constant now depends on $\theta$ as well as $n$ and $r$. 
Using this with $w=\eta H_{1}^{p / 2}$ and $r=2$, and assuming also that $n \geq 3$, we obtain

$$
\left(\int_{M_{2 \rho}}\left(\eta^{2} H_{1}^{p}\right)^{\frac{n}{n-2}}\right)^{\frac{n-2}{n}} \leq C(n, \theta)\left\{\int_{M_{2 \rho}}\left|\nabla\left(\eta H_{1}^{\frac{p}{2}}\right)\right|^{2}+\int_{M_{2 \rho}} \eta^{2} H_{1}^{p+2}\right\} .
$$

Therefore, from (2.26) we have

$$
\begin{aligned}
& 2\left(\int_{M_{2 \rho}}\left(\eta^{2} H_{1}^{p}\right)^{\frac{n}{n-2}}\right)^{\frac{n-2}{n}}+\int_{M_{2 \rho}}\left|\nabla\left(\eta H_{1}^{\frac{p}{2}}\right)\right|^{2} \\
& \leq C(n, \theta)\left\{\int_{M_{2 \rho}}\left|\nabla\left(\eta H_{1}^{\frac{p}{2}}\right)\right|^{2}+\int_{M_{2 \rho}} \eta^{2} H_{1}^{p+2}\right\} \\
& \leq C\left\{(1+p) \int_{M_{2 \rho}} \eta^{2} H_{1}^{p+2}+\frac{1+p^{2}}{\rho^{2}} \int_{M_{2 \rho}} H_{1}^{p}+\frac{1}{\rho^{2}} \int_{M_{2 \rho}} H_{1}^{p+1} H_{k-1},\right\},
\end{aligned}
$$

where $C$ depends on $k, n, \mu$ and $\theta$, but not on $p$ or $\rho$. The second term on the left hand side is included for a later purpose.

Next we remove the term $\int_{M_{2} \rho} \eta^{2} H_{1}^{p+2}$. If $k \geq 3$, the exponent $p+2$ is too large for the iteration inequality we will eventually obtain, but for $k=2$ the term causes no difficulties and can be left. Using Hölder's inequality we have

$$
\int_{M_{2 \rho}} \eta^{2} H_{1}^{p+2} \leq\left(\int_{M_{2 \rho}}\left(\eta^{2} H_{1}^{p}\right)^{\frac{n}{n-2}}\right)^{\frac{n-2}{n}}\left(\int_{M_{2 \rho}} H_{1}^{n}\right)^{\frac{2}{n}} .
$$

Therefore it is sufficient to show that

$$
\left(\int_{M_{2 \rho}} H_{1}^{n}\right)^{\frac{2}{n}} \leq \frac{1}{(1+p) C}
$$

for sufficiently small $\rho$, where $C$ is the constant in the last line of (2.29).

We have a Minkowski area bound

$$
\left|M_{2 \rho}\right|:=\mu_{L}\left(M_{2 \rho}\right) \leq C_{1} \rho^{n},
$$

and we will eventually assume that

$$
\int_{M} H_{1}^{s} \leq C_{2} \quad \text { for some } \quad s>k(n-1) / 2 .
$$

Therefore by Hölder's inequality

$$
\int_{M_{2 \rho}} H_{1}^{n} \leq\left(\int_{M_{2 \rho}} H_{1}^{s}\right)^{n / s}\left|M_{2 \rho}\right|^{1-n / s} \leq C_{2}^{n / s} C_{1}^{1-n / s} \rho^{n(1-n / s)}
$$


The exponent of $\rho$ is positive because $s>k(n-1) / 2 \geq 3(n-1) / 2 \geq n$ if $k, n \geq 3$. For (2.30) to hold we therefore need to assume that

$$
\rho \leq \rho_{0}:=\min \left\{C_{0}(1+p)^{-[2(1-n / s)]^{-1}}, \frac{\sqrt{1-\theta^{2}} d_{0}}{2}\right\}
$$

where $C_{0}$ depends on $k, n, \mu, \theta, p, s$ and $\int_{M} H_{1}^{s}$ for some $s>k(n-1) / 2$. Then we have

$$
\begin{aligned}
& \left(\int_{M_{2 \rho}} \eta^{2} H_{1}^{\frac{n p}{n-2}}\right)^{\frac{n-2}{n}}+\int_{M_{2 \rho}}\left|\nabla\left(\eta H_{1}^{\frac{p}{2}}\right)\right|^{2} \\
& \leq \frac{C}{\rho^{2}}\left\{\left(1+p^{2}\right) \int_{M_{2 \rho}} H_{1}^{p}+\int_{M_{2 \rho}} H_{1}^{p+1} H_{k-1},\right\} \\
& \leq \frac{C\left(1+p^{2}\right)}{\rho^{2}} \int_{M_{2 \rho}} H_{1}^{p+1} H_{k-1} .
\end{aligned}
$$

Here we have used the estimates

$$
H_{k}^{1 / k} \leq C_{1}(k, n) H_{k-1}^{1 /(k-1)} \leq C_{2}(k, n) H_{1}
$$

to get the last line. These inequalities are consequences of the Maclaurin inequalities ([15], Section 2.15)

$$
\left[\left(\begin{array}{c}
n \\
m
\end{array}\right)^{-1} S_{m}(\lambda)\right]^{1 / m} \leq\left[\left(\begin{array}{l}
n \\
l
\end{array}\right)^{-1} S_{l}(\lambda)\right]^{1 / l} \quad \text { for } 1 \leq l \leq m \leq n, \lambda \in \Gamma_{m} .
$$

It is convenient to state separately the two estimates implicit in (2.34). Since $\eta=1$ on $M_{\rho}$, we have

$$
\begin{aligned}
\left(\int_{M_{\rho}} H_{1}^{\frac{n p}{n-2}}\right)^{\frac{n-2}{n}} & \leq \frac{C\left(1+p^{2}\right)}{\rho^{2}} \int_{M_{2 \rho}} H_{1}^{p+1} H_{k-1} \\
& \leq \frac{C\left(1+p^{2}\right)}{\rho^{2}} \int_{M_{2 \rho}} H_{1}^{p+k}
\end{aligned}
$$

where we have again used (2.35), and

$$
\int_{M_{\rho}}\left|\nabla\left(H_{1}^{\frac{p}{2}}\right)\right|^{2} \leq \frac{C\left(1+p^{2}\right)}{\rho^{2}} \int_{M_{2 \rho}} H_{1}^{p+1} H_{k-1},
$$

both valid for $\rho>0$ satisfying (2.33).

For $k=2$ the estimates (2.37) and (2.38) are valid for all $\rho \in$ $\left(0, \sqrt{1-\theta^{2}} d_{0} / 2\right]$; the restriction $(2.33)$ is not needed.

Condition (2.33) makes it difficult to iterate (2.37) directly to get an $L^{\infty}$ bound for $H_{1}$, so we now remove this restriction. Since $M$ is strictly spacelike, for any $r \in(0,2 R]$ we have, from (2.11),

$$
c_{1} r^{n} \leq\left|M_{r}\right| \leq c_{2} r^{n}
$$


for some positive constants $c_{1}$ and $c_{2}$ depending on $n$ and $\theta$. We cover $M_{r}$ by $N$ Lorentz balls $L_{\rho}\left(X_{j}\right)$ in $\mathbf{L}^{n+1}$ of radii $\rho$ with centres $X_{j} \in M_{r}$. This can be done with

$$
N \leq C(n)(r / \rho)^{n}
$$

Then, setting $\beta=n /(n-2)$, we have

$$
\begin{aligned}
\left(\int_{M \cap L_{r}(0)} H_{1}^{\beta p}\right)^{1 / \beta} & \leq\left(\sum_{j=1}^{N} \int_{M \cap L_{\rho}\left(X_{j}\right)} H_{1}^{\beta p}\right)^{1 / \beta} \\
& \leq N^{1 / \beta}\left(\max _{1 \leq j \leq N} \int_{M \cap L_{\rho}\left(X_{j}\right)} H_{1}^{\beta p}\right)^{1 / \beta} \\
& \leq \frac{C\left(1+p^{2}\right) N^{1 / \beta}}{\rho^{2}} \int_{M \cap L_{2 r\left(X_{0}\right)}} H_{1}^{p+k} \\
& \leq \frac{C\left(1+p^{2}\right) r^{n-2}}{\rho^{n}} \int_{M \cap L_{2 r\left(X_{0}\right)}} H_{1}^{p+k}
\end{aligned}
$$

where we have used (2.40) and the fact that $\beta=n /(n-2)$ to obtain the last line. We now choose $\rho=\rho_{0}$, where $\rho_{0}$ is given by (2.33), and use (2.39) to arrive at the estimate

$$
\left(\frac{1}{\left|M_{r}\right|} \int_{M_{r}} H_{1}^{\beta p}\right)^{1 / \beta} \leq C(p+1)^{\gamma}\left(\frac{1}{\left|M_{2 r}\right|} \int_{M_{2 r}} H_{1}^{p+k}\right)
$$

where $\gamma$ is a positive constant depending on $n$ and $s$, and $C$ is independent of $p$ and $r ; C$ is independent of $r$ precisely because of (2.39) and the fact that $\beta=n /(n-2)$.

This is an inequality of exactly the same form as we obtained in [19] in the Euclidean case. We see that the exponent of integrability of $H_{1}$ improves provided $\beta p>p+k$, which reduces to $p+k>k n / 2$. A Moser type iteration starting at $p_{0}+k=s$ for some $s>k n / 2$ can be used to deduce that

$$
H_{1}(0) \leq\left(\frac{C}{r^{n}} \int_{M_{2 r}} H_{1}^{p_{0}+k}\right)^{\left[p_{0}-k n / 2\right]^{-1}},
$$

where $C$ depends only on $k, n, \mu, \theta, s$ and $\int_{M} H_{1}^{s}$ (see [19] for details). This can be viewed as a kind of mean value inequality for the mean curvature. Since $H_{1}$ is equivalent to $|A|$ for 2-admissible hypersurfaces, (2.42) implies the curvature bound stated in Theorem 1.1, under the stronger assumption $s>k n / 2$.

\section{An auxiliary estimate.}

In this section we prove an auxiliary estimate that is the key to improving the curvature bound established in Section 2. We need to recall the equation 
satisfied by $w:=\nu_{n+1}=\left(1-|D u|^{2}\right)^{-1 / 2}$ :

$$
F_{i j} \nabla_{i} \nabla_{j} w=w F_{i j} h_{i m} h_{j m}+\left\langle k g^{k-1} \nabla g, \mathbf{e}_{n+1}\right\rangle,
$$

where $\mathbf{e}_{n+1}$ is the $n+1$-st standard coordinate vector of $\mathbf{R}^{n+1}$. A proof of this is given in [19] for the Euclidean case; in the Minkowski case the proof is the same except for some sign changes, so it is omitted. For the sake of comparison, in the Euclidean case the right hand side is replaced by its negative, and of course, we also take $w=\left(1+|D u|^{2}\right)^{-1 / 2}$.

We now rotate the $x_{1}, \ldots, x_{n}$ coordinate axes so that

$$
D u(0)=|D u(0)| \mathbf{e}_{n} .
$$

We then transform $\mathbf{L}^{n+1}$ by applying a boost transformation $T$ of the form

$$
T=\left[\begin{array}{ccc}
I & 0 & 0 \\
0 & \cosh \omega & \sinh \omega \\
0 & \sinh \omega & \cosh \omega
\end{array}\right]
$$

where $D_{n} u(0)=-\tanh \omega . T$ is an isometry of $\mathbf{L}^{n+1}$. It transforms $M=$ graph $u$ to $\widetilde{M}=\operatorname{graph} \tilde{u}$ with $D \tilde{u}(0)=0$. $\tilde{u}$ is strictly spacelike, and we have

$$
\sup _{\widetilde{M}}|D \tilde{u}| \leq \tilde{\theta}<1
$$

for a controlled constant $\tilde{\theta}$ depending only on $\theta$ and $\omega$. Since $T$ is an isometry, $\tilde{u}$ is $k$-admissible and satisfies a curvature equation

$$
H_{k}[\tilde{u}]^{1 / k}=\tilde{g}(y, \tilde{u}(y))
$$

in the new coordinates $Y=T X$ with $\tilde{g}(Y)=g\left(T^{-1} Y\right)$. Furthermore, $\tilde{g}$ satisfies

$$
\tilde{\mu}^{-1} \leq \tilde{g} \leq \tilde{\mu}, \quad|\nabla \tilde{g}| \leq \tilde{\mu}
$$

for some controlled constant $\tilde{\mu}$ depending only on $\mu$ and $\omega$.

In view of this, there is no loss of generality in assuming that $D u(0)=0$, if necessary also replacing $d_{0}$ by a smaller positive constant $\tilde{d}_{0}$ depending only on $d_{0}$ and $\theta$. We therefore make this assumption in the remainder of this section. The main estimate of this section is the following.

Theorem 3.1. Let $M, u, g$ be as above with $D u(0)=0$. Then there is a constant $\rho_{1} \in\left(0, \sqrt{1-\theta^{2}} d_{0}\right]$, depending only on $k, n, p, \theta, \mu$ and the modulus of continuity of $D u$, such that for all $\rho \in\left(0, \rho_{1}\right]$ and any $p>1$ we have

$$
\int_{M_{\rho}} H_{1}^{p} H_{k-1} \leq C_{1} \rho \int_{\partial M_{\rho}} H_{1}^{p} H_{k-1}+C_{2}
$$


where $C_{1}$ depends only on $n, p$ and $\theta$, and $C_{2}$ depends on $k, n, p, \mu$ and $\theta$.

If $k=2$ we get a variant of this result without invoking the modulus of continuity of $D u$.

Theorem 3.2. Let $M, u, g$ be as above, not necessarily with $D u(0)=0$, and suppose $k=2$. Then there is a constant $\rho_{1} \in\left(0, \sqrt{1-\theta^{2}} d_{0}\right]$, depending only on $n, p, \theta$ and $\mu$, such that for all $\rho \in\left(0, \rho_{1}\right]$ and any $p>1$ we have

$$
\int_{M_{\rho}} H_{1}^{p+1} \leq C_{1} \rho \int_{\partial M_{\rho}} H_{1}^{p+1}+C_{2}
$$

where $C_{1}$ depends only on $\theta$ and $C_{2}$ depends on $n, p, \mu$ and $\theta$.

Proof of Theorem 3.1. We begin by computing a differential inequality for $W=\phi(w) H_{1}^{p}$ where $\phi$ is a smooth positive function to be chosen and $p>1$ is a constant. We have

$$
\nabla_{i} W=\phi^{\prime}(w) H_{1}^{p} \nabla_{i} w+p \phi(w) H_{1}^{p-1} \nabla_{i} H_{1}
$$

and

$$
\begin{aligned}
\nabla_{i} \nabla_{j} W= & \phi^{\prime}(w) H_{1}^{p} \nabla_{i} \nabla_{j} w+\phi^{\prime \prime}(w) H_{1}^{p} \nabla_{i} w \nabla_{j} w \\
& +p \phi^{\prime}(w) H_{1}^{p-1}\left(\nabla_{i} w \nabla_{j} H_{1}+\nabla_{j} w \nabla_{i} H_{1}\right) \\
& +p \phi(w) H_{1}^{p-1} \nabla_{i} \nabla_{j} H_{1}+p(p-1) \phi(w) H_{1}^{p-2} \nabla_{i} H_{1} \nabla_{j} H_{1} .
\end{aligned}
$$

Consequently, using (3.1) and (2.9) we have

$$
\begin{aligned}
F_{i j} \nabla_{i} \nabla_{j} W & \geq \phi^{\prime}(w) H_{1}^{p}\left\{w F_{i j} h_{i m} h_{j m}+\left\langle k g^{k-1} \nabla g, \mathbf{e}_{n+1}\right\rangle\right\} \\
& +\phi^{\prime \prime}(w) H_{1}^{p} F_{i j} \nabla_{i} w \nabla_{j} w+2 p \phi^{\prime}(w) H_{1}^{p-1} F_{i j} \nabla_{i} w \nabla_{j} H_{1} \\
& +p \phi(w) H_{1}^{p-1}\left\{H_{1} F_{i j} h_{i m} h_{j m}-F_{i j} h_{i j} h_{l m} h_{l m}+k g^{k-1} \Delta g\right\} \\
& +p(p-1) \phi(w) H_{1}^{p-2} F_{i j} \nabla_{i} H_{1} \nabla_{j} H_{1}
\end{aligned}
$$

For any $\epsilon>0$ we now estimate

$$
\begin{aligned}
& 2 p \phi^{\prime}(w) H_{1}^{p-1} F_{i j} \nabla_{i} w \nabla_{j} H_{1} \\
& \geq-\frac{p\left(\phi^{\prime}\right)^{2}}{\epsilon(p-1) \phi} H_{1}^{p} F_{i j} \nabla_{i} w \nabla_{j} w-\epsilon p(p-1) \phi H_{1}^{p-2} F_{i j} \nabla_{i} H_{1} \nabla_{j} H_{1} .
\end{aligned}
$$

Using this in (3.4) and rearranging terms we obtain

$$
\begin{aligned}
F_{i j} \nabla_{i} \nabla_{j} W \geq & H_{1}^{p} F_{i j} h_{i m} h_{j m}\left(\phi^{\prime} w+p \phi\right)-k p g^{k} \phi H_{1}^{p+1} \\
& +H_{1}^{p} F_{i j} \nabla_{i} w \nabla_{j} w\left(\phi^{\prime \prime}-\frac{p\left(\phi^{\prime}\right)^{2}}{\epsilon(p-1) \phi}\right) \\
& +(1-\epsilon) p(p-1) \phi H_{1}^{p-2} F_{i j} \nabla_{i} H_{1} \nabla_{j} H_{1} \\
& +\phi^{\prime} H_{1}^{p}\left\langle k g^{k-1} \nabla g, \mathbf{e}_{n+1}\right\rangle+k p \phi H_{1}^{p-1} g^{k-1} \Delta g
\end{aligned}
$$


where we have used the estimate

$$
F_{i j} h_{i j} h_{l m} h_{l m}=k g^{k}|A|^{2} \leq k g^{k} H_{1}^{2} .
$$

To obtain the last inequality we have used the 2-admissibility of $M$.

Next we estimate $F_{i j} h_{i m} h_{j m}$ from below. We denote the eigenvalues of $\left[h_{i j}\right]$ by $\lambda_{1}, \ldots, \lambda_{n}$ and let

$$
S_{k-1 ; i}(\lambda)=\frac{\partial S_{k}(\lambda)}{\partial \lambda_{i}}=\left.S_{k}(\lambda)\right|_{\lambda_{i}=0} .
$$

Then, since

$$
S_{k}(\lambda)=S_{k-1 ; i}(\lambda)+S_{k ; i}(\lambda) \quad \text { for each } i=1, \ldots, n,
$$

we have

$$
\begin{aligned}
F_{i j} h_{i m} h_{j m} & =\sum_{i=1}^{n} S_{k-1 ; i}(\lambda) \lambda_{i}^{2} \\
& =\sum_{i=1}^{n} S_{k}(\lambda) \lambda_{i}-\sum_{i=1}^{n} S_{k ; i}(\lambda) \lambda_{i} \\
& =S_{k}(\lambda) S_{1}(\lambda)-(k+1) S_{k+1}(\lambda) \\
& \geq \frac{k}{n} S_{k}(\lambda) S_{1}(\lambda) \\
& =\frac{k}{n} H_{k} H_{1},
\end{aligned}
$$

where we take $S_{k+1}=H_{k+1}=0$ if $k=n$. To obtain the second last line we have used the inequality

$$
\frac{S_{l-1}(\lambda)}{\left(\begin{array}{c}
n \\
l-1
\end{array}\right)} \frac{S_{m}(\lambda)}{\left(\begin{array}{c}
n \\
m
\end{array}\right)} \leq \frac{S_{l}(\lambda)}{\left(\begin{array}{l}
n \\
l
\end{array}\right)} \frac{S_{m-1}(\lambda)}{\left(\begin{array}{c}
n \\
m-1
\end{array}\right)}
$$

which is valid for $\lambda \in \Gamma_{m}$ and $0 \leq l<m \leq n$. This follows easily from the Newton inequalities ([15], Section 2.15)

$$
\frac{S_{r-1}(\lambda)}{\left(\begin{array}{c}
n \\
r-1
\end{array}\right)} \frac{S_{r+1}(\lambda)}{\left(\begin{array}{c}
n \\
r+1
\end{array}\right)} \leq\left(\frac{S_{r}(\lambda)}{\left(\begin{array}{c}
n \\
r
\end{array}\right)}\right)^{2} \quad \text { for all } \quad \lambda \in \mathbf{R}^{n}, \quad r=1, \ldots, n .
$$

It follows from (3.8) that

$$
\begin{aligned}
& H_{1}^{p} F_{i j} h_{i m} h_{j m}\left[\phi^{\prime} w+p \phi\right]-k p g^{k} \phi H_{1}^{p+1} \\
& \geq \frac{1}{2} H_{1}^{p} F_{i j} h_{i m} h_{j m}\left[\phi^{\prime} w+p \phi\right]+\frac{k}{2 n} g^{k} H_{1}^{p+1}\left[\phi^{\prime} w+p \phi-2 n p \phi\right] .
\end{aligned}
$$

We now set

$$
\phi(w)=w^{q}
$$

for $q>0$ to be chosen. Then

$$
\phi^{\prime} w+p \phi-2 n p \phi \geq[q-(2 n-1) p] w^{q} \geq \frac{1}{2} q w^{q} \quad \text { if } \quad q \geq 2(2 n-1) p .
$$


Furthermore

$$
\phi^{\prime \prime}=q(q-1) w^{q-2}
$$

Next we have

$$
\frac{p\left(\phi^{\prime}\right)^{2}}{\epsilon(p-1) \phi}=\frac{p q^{2}}{\epsilon(p-1)} w^{q-2}
$$

Since we will eventually fix $\epsilon \in(0,1)$, it is apparent then that (3.12) cannot be controlled from above by (3.11) for all $p>1$. Instead we control the term

$$
\frac{p\left(\phi^{\prime}\right)^{2}}{\epsilon(p-1) \phi} H_{1}^{p} F_{i j} \nabla_{i} w \nabla_{j} w
$$

using the first term on the left hand side of (3.9).

To do this we recall that $h_{i j}=\left\langle D_{\hat{\mathbf{e}}_{i}} \nu, \hat{\mathbf{e}}_{j}\right\rangle$, where $D$ is the connection on $\mathbf{L}^{n+1}$. Using the facts that $\left\langle\hat{\mathbf{e}}_{k}, \nu\right\rangle=0$ and $\left\langle D_{\hat{\mathbf{e}}_{i}} \nu, \nu\right\rangle=0$, and denoting the standard $n+1$-st coordinate vector in $\mathbf{R}^{n+1}$ by $\mathbf{e}_{n+1}$, we compute

$$
\nabla_{i} w=-\left\langle D_{\hat{\mathbf{e}}_{i}} \nu, \mathbf{e}_{n+1}\right\rangle=-\left\langle D_{\hat{\mathbf{e}}_{i}} \nu, \hat{\mathbf{e}}_{j}\right\rangle\left\langle\hat{\mathbf{e}}_{j}, \mathbf{e}_{n+1}\right\rangle=-h_{i j}\left\langle\hat{\mathbf{e}}_{j}, \mathbf{e}_{n+1}\right\rangle .
$$

$\hat{\mathbf{e}}_{k}$ is a unit tangent vector field to $M=\operatorname{graph} u$, so for each $k=1, \ldots, n$ there is a unit vector field $\xi_{k}$ defined near $0 \in \mathbf{R}^{n}$ such that

$$
\hat{\mathbf{e}}_{k}=\frac{\left(\xi_{k}, D_{\xi_{k}} u\right)}{\sqrt{1-\left|D_{\xi_{k}} u\right|^{2}}} .
$$

Then

$$
\left\langle\hat{\mathbf{e}}_{k}, \mathbf{e}_{n+1}\right\rangle=\frac{D_{\xi_{k}} u}{\sqrt{1-\left|D_{\xi_{k}} u\right|^{2}}}
$$

and consequently

$$
\begin{aligned}
F_{i j} \nabla_{i} w \nabla_{j} w & =F_{i j} h_{i k} h_{j l} \frac{D_{\xi_{k}} u D_{\xi_{l}} u}{\sqrt{1-\left|D_{\xi_{k}} u\right|^{2}} \sqrt{1-\left|D_{\xi_{l}} u\right|^{2}}} \\
& \leq|D u|^{2} w^{2} F_{i j} h_{i k} h_{j k} .
\end{aligned}
$$

Thus, using (3.12) and (3.13),

$$
\begin{aligned}
\frac{p\left(\phi^{\prime}\right)^{2}}{\epsilon(p-1) \phi} H_{1}^{p} F_{i j} \nabla_{i} w \nabla_{j} w & \leq \frac{p q^{2}}{\epsilon(p-1)}|D u|^{2} w^{q} H_{1}^{p} F_{i j} h_{i k} h_{j k} \\
& \leq \frac{1}{2} q w^{q} H_{1}^{p} F_{i j} h_{i k} h_{j k}
\end{aligned}
$$

provided

$$
\frac{p q}{\epsilon(p-1)}|D u|^{2} \leq \frac{1}{2}
$$


We now fix $\epsilon=1 / 2$ and set $q=2(2 n-1) p$, so that $(3.10)$ is valid. Then (3.14) holds whenever

$$
|D u|^{2} \leq \frac{p-1}{8(2 n-1) p^{2}}
$$

And this is clearly valid for all $X \in M_{\bar{\rho}}=M \cap L_{\bar{\rho}}$ with sufficiently small $\bar{\rho}>0$, depending only $n, p$ and the modulus of continuity of $D u$.

Combining the above estimates we see that for any $p>1$ we have

$$
\begin{aligned}
F_{i j} \nabla_{i} \nabla_{j} W \geq & \frac{k q}{4 n} w^{q} g^{k} H_{1}^{p+1}+q(q-1) w^{q-2} H_{1}^{p} F_{i j} \nabla_{i} w \nabla_{j} w \\
& +\frac{1}{2} p(p-1) w^{q} H_{1}^{p-2} F_{i j} \nabla_{i} H_{1} \nabla_{j} H_{1} \\
& +q w^{q-1} H_{1}^{p}\left\langle k g^{k-1} \nabla g, \mathbf{e}_{n+1}\right\rangle+k p w^{q} H_{1}^{p-1} g^{k-1} \Delta g
\end{aligned}
$$

in $M_{\bar{\rho}}$, for sufficiently small $\bar{\rho}>0$ depending only on $n, p$ and the modulus of continuity of $D u$.

We now set $\eta(X)=\rho^{2}-\langle X, X\rangle=\rho^{2}-|l(X)|^{2}$ where $\rho \in(0, \bar{\rho}]$. Let $M_{\rho}=$ $M \cap L_{\rho}$. Then $\partial M_{\rho}=M \cap \partial L_{\rho}$ because $M$ and $\partial L_{\rho}$ intersect transversally, since $M$ is a strictly spacelike graph. We now multiply (3.16) by $\eta$ and integrate over $M_{\rho}$. Integrating by parts twice and using the fact that $F_{i j}$ is divergence free (see (2.4)) we obtain

$$
\begin{aligned}
& \quad \frac{k q}{4 n} \int_{M_{\rho}} \eta w^{q} g^{k} H_{1}^{p+1}+q(q-1) \int_{M_{\rho}} \eta w^{q} H_{1}^{p} F_{i j} \nabla_{i} w \nabla_{j} w \\
& \quad+\frac{1}{2} p(p-1) \int_{M_{\rho}} \eta w^{q} H_{1}^{p-2} F_{i j} \nabla_{i} H_{1} \nabla_{j} H_{1} \\
& \quad+q \int_{M_{\rho}} \eta w^{q-1} H_{1}^{p}\left\langle k g^{k-1} \nabla g, \mathbf{e}_{n+1}\right\rangle+k p \int_{M_{\rho}} \eta w^{q} H_{1}^{p-1} g^{k-1} \Delta g \\
& \leq \int_{M_{\rho}} \eta F_{i j} \nabla_{i} \nabla_{j} W \\
& =-\int_{M_{\rho}} F_{i j} \nabla_{i} \eta \nabla_{j} W \\
& =\int_{M_{\rho}} W F_{i j} \nabla_{i} \nabla_{j} \eta-\int_{\partial M_{\rho}} W F_{i j} \nabla_{i} \eta \mathcal{N}_{j}
\end{aligned}
$$

where $\mathcal{N}$ denotes the outer unit normal to $\partial M_{\rho}$ in $M$.

We now proceed to estimate the last two integrals on the left hand side of (3.17) almost exactly as in Section 2. Using the bounds $1 \leq w \leq C(n, p)$ on $M_{\rho}$ as appropriate without further mention, and the fact that $|\nabla \eta| \leq$ $2 l|\nabla l| \leq C(\theta) \rho$, we obtain

$$
\begin{aligned}
- & \int_{M_{\rho}} W F_{i j} \nabla_{i} \nabla_{j} \eta+\int_{\partial M_{\rho}} W F_{i j} \nabla_{i} \eta \mathcal{N}_{j} \\
\leq & -C_{1} \int_{M_{\rho}} \eta g^{k} H_{1}^{p+1}+C_{2} \int_{M_{\rho}} \eta H_{1}^{p} g^{k-1}|\nabla g| \\
& +C_{3} \int_{M_{\rho}} H_{1}^{p-1}\left(g^{k}+\rho^{2} g^{k-2}|\nabla g|^{2}\right) \\
\leq & C \int_{M_{\rho}} H_{1}^{p-1}\left(g^{k}+\rho^{2} g^{k-2}|\nabla g|^{2}\right),
\end{aligned}
$$

where $C_{1}, C_{2}, C_{3}$ and $C$ are positive constants depending only on $k, n, \theta$ and $p$. 
Next we compute the derivatives of $\eta$. Using Gauss's formula

$$
\nabla_{i} \nabla_{j} X_{k}=h_{i j} \nu_{k}
$$

we compute

$$
\begin{aligned}
\nabla_{i} \nabla_{j} \eta & =-2\left\langle\nabla_{i} X, \nabla_{j} X\right\rangle-2\left\langle X, \nabla_{i} \nabla_{j} X\right\rangle \\
& =-2 \delta_{i j}-2 h_{i j}\langle X, \nu\rangle .
\end{aligned}
$$

Consequently

$$
\begin{aligned}
F_{i j} \nabla_{i} \nabla_{j} \eta & =-2 \sum_{i=1}^{n} F_{i i}-2 F_{i j} h_{i j}\langle X, \nu\rangle \\
& =-2(n-k+1) H_{k-1}-2 k g^{k}\langle X, \nu\rangle .
\end{aligned}
$$

In addition, on $\partial M_{\rho}$ we have

$$
\begin{aligned}
-F_{i j} \nabla_{i} \eta \mathcal{N}_{j} & =2 l F_{i j} \nabla_{i} l \mathcal{N}_{j} \\
& =2 l F_{i j} \frac{\nabla_{i} l}{|\nabla l|} \mathcal{N}_{j} \\
& \leq 2 l|\nabla l| \sum_{i=1}^{n} F_{i i} \\
& =2(n-k+1) \rho|\nabla l| H_{k-1}
\end{aligned}
$$

where we have used (2.14) and (2.17). Using (3.20) and (3.21) in (3.18) and estimating the last term in (3.20) in an obvious way we obtain

$$
\begin{aligned}
& \int_{M_{\rho}} w^{q} H_{1}^{p} H_{k-1} \leq \rho \int_{\partial M_{\rho}}|\nabla l| w^{q} H_{1}^{p} H_{k-1} \\
& \quad+C_{1} \rho \int_{M_{\rho}} g^{k} H_{1}^{p}+C_{2} \int_{M_{\rho}} H_{1}^{p-1}\left(g^{k}+\rho^{2} g^{k-2}|\nabla g|^{2}\right)
\end{aligned}
$$

for any $\rho \in(0, \bar{\rho}]$, where $C_{1}$ and $C_{2}$ depend only on $k, n, p$ and $\theta$, and $q=$ $2(2 n-1) p$. We then estimate $w$ from above and below as before and estimate $|\nabla l|$ using (2.14). Since $g \leq \mu$, and $H_{1}, H_{k-1}$ are bounded away from zero by a positive constant depending only on $k, n$ and $\mu$, for $\rho$ sufficiently small, say $\rho \in\left(0, \rho_{1}\right]$, the second and third terms on the right hand side of (3.22) can be absorbed into the left, at the expense of leaving a constant on the right, and we deduce the estimate of Theorem 3.1.

Proof of Theorem 3.2. To prove Theorem 3.2 we proceed as before, but now we take $\phi=1$. We compute

$$
\begin{aligned}
F_{i j} \nabla_{i} \nabla_{j} W \geq & p H_{1}^{p-1}\left\{H_{1} F_{i j} h_{i m} h_{j m}-F_{i j} h_{i j} h_{l m} h_{l m}+k g^{k-1} \Delta g\right\} \\
& +p(p-1) H_{1}^{p-2} F_{i j} \nabla_{i} H_{1} \nabla_{j} H_{1} \\
\geq & -k p g^{k} H_{1}^{p+1}+k p H_{1}^{p-1} g^{k-1} \Delta g \\
& +p(p-1) H_{1}^{p-2} F_{i j} \nabla_{i} H_{1} \nabla_{j} H_{1} .
\end{aligned}
$$


For the same choice of $\eta$ as above this leads to the estimate

$$
\begin{aligned}
& \int_{M_{\rho}} H_{1}^{p} H_{k-1} \leq \rho \int_{\partial M_{\rho}}|\nabla l| H_{1}^{p} H_{k-1}+C_{1} \rho^{2} \int_{M_{\rho}} g^{k} H_{1}^{p+1} \\
& \quad+C_{2} \rho \int_{M_{\rho}} g^{k} H_{1}^{p}+C_{3} \int_{M_{\rho}} H_{1}^{p-1}\left(g^{k}+\rho^{2} g^{k-2}|\nabla g|^{2}\right)
\end{aligned}
$$

for any $\rho \in\left(0, \sqrt{1-\theta^{2}} d_{0}\right]$, where $C_{1}, C_{2}, C_{3}$ depend only on $k, n, p$ and $\theta$. We observe that this estimate is valid for any $k=2, \ldots, n$ and any $p>1$, without requiring $\rho$ to be small. We now obtain Theorem 3.2 by taking $k=2$ and then requiring $\rho$ to be so small that the last three terms on the right hand side of (3.24) can be absorbed into the left, at the expense of leaving a constant on the right, as before. This completes the proof of Theorem 3.2. Remark If $g$ is constant, the last term in (3.22) and (3.24) can be dropped.

To end this section we describe some further results that can be obtained from the above estimates. These are not needed for the proof of Theorem 1.1, but we believe they are nevertheless of some interest.

From (3.22) and (3.24) we can derive monotonicity formulae analogous to those established in [20] in the Euclidean case. By the coarea formula ([16], Chapter 10 or [9], Theorem 3.2.22) we have

$$
\begin{aligned}
\int_{\partial M_{\rho}}|\nabla l| w^{q} H_{1}^{p} H_{k-1} & \leq C(\theta) \int_{\partial M_{\rho}} \frac{w^{q} H_{1}^{p} H_{k-1}}{|\nabla l|} \\
& =C(\theta) \frac{d}{d \rho} \int_{M_{\rho}} w^{q} H_{1}^{p} H_{k-1}
\end{aligned}
$$

where

$$
C(\theta)=\sup _{M}|\nabla l|^{2} \geq 1
$$

Setting $\beta=1 / C(\theta)$ and using (3.25) we can rewrite (3.22) as

$$
\begin{aligned}
& -\frac{d}{d \rho}\left(\frac{1}{\rho^{\beta}} \int_{M_{\rho}} w^{q} H_{1}^{p} H_{k-1}\right) \\
& \leq \frac{C}{\rho^{1+\beta}}\left\{\rho \int_{M_{\rho}} g^{k} H_{1}^{p}+\int_{M_{\rho}} H_{1}^{p-1}\left(g^{k}+\rho^{2} g^{k-2}|\nabla g|^{2}\right)\right\} .
\end{aligned}
$$

Integrating this from $r$ to $R$ where $0<r \leq R \leq \bar{\rho}$, we obtain the monotonicity formula

$$
\begin{aligned}
& \frac{1}{r^{\beta}} \int_{M_{r}} w^{q} H_{1}^{p} H_{k-1} \leq \frac{1}{R^{\beta}} \int_{M_{R}} w^{q} H_{1}^{p} H_{k-1} \\
& +C \int_{r}^{R} \frac{1}{\rho^{1+\beta}}\left\{\rho \int_{M_{\rho}} g^{k} H_{1}^{p}+\int_{M_{\rho}} H_{1}^{p-1}\left(g^{k}+\rho^{2} g^{k-2}|\nabla g|^{2}\right)\right\} d \rho,
\end{aligned}
$$

where in (3.26) and (3.27) $C$ depends only on $k, n, p$ and $\theta$, and $p>1$, $q=2(2 n-1) p$. 
In a similar way, from (3.24) we obtain, for $0<r \leq R \leq \sqrt{1-\theta^{2}} d_{0}$,

$$
\begin{aligned}
& \frac{1}{r^{\beta}} \int_{M_{r}} H_{1}^{p} H_{k-1} \leq \frac{1}{R^{\beta}} \int_{M_{R}} H_{1}^{p} H_{k-1} \\
& \quad+C \int_{r}^{R} \frac{1}{\rho^{1+\beta}}\left\{\rho^{2} \int_{M_{\rho}} g^{k} H_{1}^{p+1}+\rho \int_{M_{\rho}} g^{k} H_{1}^{p}\right. \\
& \left.\quad+\int_{M_{\rho}} H_{1}^{p-1}\left(g^{k}+\rho^{2} g^{k-2}|\nabla g|^{2}\right)\right\} d \rho .
\end{aligned}
$$

It is of course possible to restrict $r, R$ so that $0<r \leq R \leq \rho_{1}$, and obtain simpler monotonicity formulae from the inequalities (3.2) and (3.3), rather than from (3.22) and (3.24). We state only the version arising from (3.3), since this is the most interesting.

Theorem 3.3. Let $M, u, g$ be as in Theorem 3.2, with $k=2$. Then for any $q>2$ there exists $\rho_{1} \in\left(0, \sqrt{1-\theta^{2}} d_{0}\right]$, depending only on $n, q, \mu$ and $\theta$, such that for any $0<r \leq R \leq \rho_{1}$ we have

$$
\frac{1}{r^{\beta}} \int_{M_{r}} H_{1}^{q} \leq \frac{1}{R^{\beta}} \int_{M_{R}} H_{1}^{q}+\frac{C}{\beta r^{\beta}}
$$

where $C$ depends only on $n, q, \mu$ and $\theta$, and

$$
\beta=\beta(\theta)=\left(\sup _{M}|\nabla l|^{2}\right)^{-1} \leq 1 .
$$

An interesting corollary of this is the following local Hölder gradient estimate for the case $k=2$. It follows directly from Theorem 3.3 and Morrey's Lemma.

Theorem 3.4. Let $M, u, g, \rho_{1}, \beta$ be as in Theorem 3.3. Then for any $q>$ $n-\beta, n \geq 3$, and any $r \in\left(0, \rho_{1} / 4\right]$ we have an estimate

$$
\sup _{\substack{x, y \in B_{r} \\ x \neq y}} \frac{|D u(x)-D u(y)|}{|x-y|^{\alpha}} \leq C r^{-1 / q}\left(\int_{M_{\rho_{1}}} H_{1}^{q}+1\right),
$$

where $\alpha=1-(n-\beta) / q$ and $C$ depends only on $n, q, \mu$ and $\theta$.

Remarks. (i) It is apparent from the estimates above that Theorems 3.3 and 3.4 are valid under the weaker assumptions

$$
0 \leq g \leq \mu, \quad|\nabla g| \leq \mu \quad \text { on } \quad M
$$

i.e., we can allow the equation to degenerate. 
(ii) Once we have a modulus of continuity estimate for $D u$, the constant $\beta$ in (3.30) can be made as close to 1 as we like by first applying a boost transformation to reduce to the case $D u(0)=0$ as in the beginning of this section, and then restricting to a small enough neighbourhood of 0 to make $\theta$ as small as required. We can then deduce $(3.31)$ with any $\beta \in(0,1)$ and with $r \in\left(0, \rho_{2}\right]$ for some $\rho_{2}>0$ such that $\rho_{2} \rightarrow 0$ as $\beta \rightarrow 1$.

(iii) A result analogous to Theorem 3.4 was proved in the Euclidean case [20] with $\beta=1$; in that case we also had to assume a modulus of continuity estimate for $D u$.

\section{An improved interior curvature bound.}

In this section we will use the estimate of Theorems 2.1 and 2.2 to improve the interior curvature estimate of Section 2. As in the Euclidean case [20], the key idea is to use this to derive a better iteration inequality than that obtained in Section 2. This part of the argument closely follows the Euclidean case and is included for completeness and the convenience of the reader.

We will use the Sobolev inequality of Allard [1] and Michael and Simon [14], but it will be applied a little differently than in Section 2. We will use it in the following form: for any smooth submanifold $\Sigma \subset \mathbf{R}^{n+1}$ of dimension $n-1$, any function $w \in C_{0}^{1}(\Sigma)$ and any $r \in[1, n-1)$ we have

$$
\begin{aligned}
& \left(\int_{\Sigma}|w|^{(n-1) r /(n-1-r)} d \sigma\right)^{(n-1-r) /(n-1) r} \\
& \leq C(n, r)\left(\int_{\Sigma}\left|\nabla^{\Sigma} w\right|^{r} d \sigma+\int_{\Sigma}\left|\mathbf{H}_{\Sigma} w\right|^{r} d \sigma\right)^{1 / r},
\end{aligned}
$$

where $\mathbf{H}_{\Sigma}$ denotes the mean curvature vector of $\Sigma, \nabla^{\Sigma}$ denotes the tangential gradient operator on $\Sigma$, and $\sigma$ denotes the $n$-1-dimensional measure on $\Sigma$.

Let us assume for the moment that $n \geq 4$; we will indicate the modifications that need to be made in the case $n=3$ later. We first consider $M$ with the metric induced by the Euclidean metric on $\mathbf{R}^{n} \times \mathbf{R}$, and apply (4.1) with $\Sigma=\Sigma_{t}=M \cap \partial L_{t}, t \in[\rho / 2, \rho], w=H_{1}^{q / 2}$ and $r=2$ to get

$$
\begin{aligned}
& \left(\int_{\Sigma_{t}} H_{1}^{\frac{q(n-1)}{n-3}} d \sigma_{E}\right)^{\frac{n-3}{n-1}} \\
& \leq C(n)\left\{\int_{\Sigma_{t}}\left|\nabla_{E}^{\Sigma_{t}}\left(H_{1}^{\frac{q}{2}}\right)\right|^{2} d \sigma_{E}+\int_{\Sigma_{t}}\left|\mathbf{H}_{\Sigma_{t}}^{E}\right|^{2} H_{1}^{q} d \sigma_{E}\right\} \\
& \leq C\left\{\int_{\Sigma_{t}}\left|\nabla_{E}\left(H_{1}^{\frac{q}{2}}\right)\right|^{2} d \sigma_{E}+\rho^{-2} \int_{\Sigma_{t}} H_{1}^{q} d \sigma_{E}+\int_{\Sigma_{t}} H_{1}^{q+2} d \sigma_{E}\right\},
\end{aligned}
$$

with $C$ independent of $\rho$. Here $\nabla_{E}^{\Sigma_{t}}$ and $\nabla_{E}$ denote the connections on $\Sigma_{t}$ and $M$ respectively induced by the Euclidean metric on $\mathbf{R}^{n} \times \mathbf{R}$ and $\sigma_{E}$ 
denotes the corresponding $n$-1-dimensional measure on $\Sigma_{t}$. In particular, $\left|\nabla_{E}^{\Sigma_{t}} f\right| \leq\left|\nabla_{E} f\right|$ for any smooth function $f$ on $M$. To obtain the last two terms in (3.2) we have used the fact that

$$
\left|\mathbf{H}_{\Sigma_{t}}^{E}\right| \leq C\left(\rho^{-1}+H_{M}^{E}\right) \leq C\left(\rho^{-1}+H_{1}\right),
$$

because $t \in[\rho / 2, \rho]$, and because $M$ and $\partial L_{t}$ have intersection angle bounded away from zero at each point of intersection, since $M$ is strictly spacelike (see [20], Lemma 3.1). As before, Euclidean and Minkowski quantities are comparable, since $M$ is strictly spacelike. In addition, $\left|\nabla_{L}^{\Sigma_{t}} f\right| \leq\left|\nabla_{L} f\right|$ for any smooth function $f$ on $M$. Consequently, from (4.2) we obtain

$$
\begin{aligned}
& \left(\int_{\Sigma_{t}} H_{1}^{\frac{q(n-1)}{n-3}} d \sigma_{L}\right)^{\frac{n-3}{n-1}} \\
& \leq C(n, \theta)\left\{\int_{\Sigma_{t}}\left|\nabla_{L}\left(H_{1}^{\frac{q}{2}}\right)\right|^{2} d \sigma_{L}+\rho^{-2} \int_{\Sigma_{t}} H_{1}^{q} d \sigma_{L}+\int_{\Sigma_{t}} H_{1}^{q+2} d \sigma_{L}\right\} .
\end{aligned}
$$

For the remainder of this section we drop the subscripts and superscripts $L$ and omit the measure $\sigma_{L}$. All integrals from now on will be with respect to the measures induced by the Minkowski metric, and it will be clear whether an integral over an $n$-dimensional or $n$-1-dimensional set is intended.

Next we need to deal with the term $\int_{\Sigma_{t}} H_{1}^{q+2}$ in (4.4). If this term is left as it is, the iteration inequality that we eventually obtain has a term $\int_{M_{4 \rho}} H_{1}^{q+2}$ on the right hand side, which causes the iteration procedure to break down if $k \geq 3$. For $k=2$, however, this term causes no difficulties. We will now show that for $k \geq 3$, the term in question can be absorbed into the left hand side of (4.4) for a sufficiently large set of $t \in[\rho / 2, \rho]$. This will be sufficient for the proof.

By Hölder's inequality we have

$$
\int_{\Sigma_{t}} H_{1}^{q+2} \leq\left(\int_{\Sigma_{t}} H_{1}^{\frac{q(n-1)}{n-3}}\right)^{\frac{n-3}{n-1}}\left(\int_{\Sigma_{t}} H_{1}^{n-1}\right)^{\frac{2}{n-1}} .
$$

Consequently it is sufficient to show that

$$
\left(\int_{\Sigma_{t}} H_{1}^{n-1}\right)^{\frac{2}{n-1}} \leq \frac{1}{2 C}
$$

where $C$ is the constant from (4.4).

We will use the following two facts. First, since $M_{\rho}$ is a strictly spacelike graph, we have the Minkowski area bound

$$
\left|M_{t}\right| \leq C_{1} \rho^{n}
$$


for each $t \in(0, \rho]$. Second, we are assuming that for some $s>k(n-1) / 2>$ $n-1$ we have

$$
\int_{M_{\rho}} H_{1}^{s} \leq C_{2}
$$

By the coarea formula ([16], Chapter 10 or [9], Theorem 3.2.22) and the fact that $|\nabla l| \leq C(\theta)$ we have

$$
\int_{\rho / 2}^{\rho} \sigma\left(\Sigma_{t}\right) d t=\int_{M_{\rho}-M_{\rho / 2}}|\nabla l| \leq C(\theta)\left|M_{\rho}\right| \leq C_{3} \rho^{n} .
$$

Thus for any $\epsilon \in(0,1)$ we have

$$
\sigma\left(\Sigma_{t}\right) \leq \frac{2 C_{3} \rho^{n-1}}{\epsilon}
$$

for $t$ belonging to a subset $I=I(\epsilon) \subset[\rho / 2, \rho]$ of measure at least $(1-\epsilon) \rho / 2$. By the coarea inequality again we have

$$
\int_{\rho / 2}^{\rho}\left(\int_{\Sigma_{t}} H_{1}^{s}\right) d t=\int_{M_{\rho}-M_{\rho / 2}}|\nabla l| H_{1}^{s} \leq C(\theta) \int_{M_{\rho}} H_{1}^{s} \leq C_{4} .
$$

Therefore for any $\epsilon^{\prime} \in(0,1)$ we have

$$
\int_{\Sigma_{t}} H_{1}^{s} \leq \frac{2 C_{4}}{\epsilon^{\prime} \rho}
$$

for $t$ belonging to a subset $J=J\left(\epsilon^{\prime}\right) \subset[\rho / 2, \rho]$ of measure at least $\left(1-\epsilon^{\prime}\right) \rho / 2$.

Let us now fix $\epsilon=\epsilon^{\prime}=1 / 8$. Then $|I \cap J| \geq 3 \rho / 8$, and for $t \in I \cap J$ we have, by Hölder's inequality and (4.9), (4.10),

$$
\begin{aligned}
\int_{\Sigma_{t}} H_{1}^{n-1} & \leq\left(\int_{\Sigma_{t}} H_{1}^{s}\right)^{\frac{n-1}{s}}\left(\sigma\left(\Sigma_{t}\right)\right)^{1-\frac{n-1}{s}} \\
& \leq\left(\frac{16 C_{4}}{\rho}\right)^{\frac{n-1}{s}}\left(16 C_{3} \rho^{n-1}\right)^{1-\frac{n-1}{s}} \\
& =C_{5} \rho^{(n-1)\left(1-\frac{n}{s}\right)} .
\end{aligned}
$$

The exponent of $\rho$ in the last line is positive because $s>k(n-1) / 2$ and $k \geq 3$. Therefore (3.6) follows for sufficiently small $\rho$, for all $t \in I \cap J \subset[\rho / 2, \rho]$.

Returning to (4.4), we now have, for $k \geq 3$ and for sufficiently small $\rho$,

$$
\left(\int_{\Sigma_{t}} H_{1}^{\frac{q(n-1)}{n-3}}\right)^{\frac{n-3}{n-1}} \leq C\left\{\int_{\Sigma_{t}}\left|\nabla\left(H_{1}^{\frac{q}{2}}\right)\right|^{2}+\int_{\Sigma_{t}} H_{1}^{q}\right\}
$$


for all $t \in I \cap J \subset[\rho / 2, \rho]$, where now the constant $C$ depends also on $\rho$. For $k=2$ we have instead

$$
\left(\int_{\Sigma_{t}} H_{1}^{\frac{q(n-1)}{n-3}}\right)^{\frac{n-3}{n-1}} \leq C\left\{\int_{\Sigma_{t}}\left|\nabla\left(H_{1}^{\frac{q}{2}}\right)\right|^{2}+\int_{\Sigma_{t}} H_{1}^{q+2}\right\},
$$

for all $t \in[\rho / 2, \rho]$.

Let us now assume that $k \geq 3$. By Hölder's inequality, the estimate (2.35) and Young's inequality, for any $p>0$ we have

$$
\begin{aligned}
\int_{\Sigma_{t}} H_{1}^{p} H_{k-1} & \leq\left(\int_{\Sigma_{t}} H_{1}^{\frac{p(q+k)}{q+1}}\right)^{\frac{q+1}{q+k}}\left(\int_{\Sigma_{t}} H_{k-1}^{\frac{q+k}{k-1}}\right)^{\frac{k-1}{q+k}} \\
& \leq C\left\{\int_{\Sigma_{t}} H_{1}^{\frac{p(q+k)}{q+1}}+\int_{\Sigma_{t}} H_{1}^{q+1} H_{k-1}\right\} .
\end{aligned}
$$

Applying this with

$$
p=\frac{q(q+1)(n-1)}{(q+k)(n-3)}>1
$$

(this condition will be satisfied by our eventual choice of $q$ ) and using (3.2) and (4.11) we get, for all $t \in I \cap J$,

$$
\begin{aligned}
& \int_{M_{t}} H_{1}^{\frac{q(q+1)(n-1)}{(q+k)(n-3)}} H_{k-1} \\
& \leq \int_{\Sigma_{t}} H_{1}^{\frac{q(q+1)(n-1)}{(q+k)(n-3)}} H_{k-1}+C \\
& \leq C\left\{\int_{\Sigma_{t}} H_{1}^{\frac{q(n-1)}{n-3}}+\int_{\Sigma_{t}} H_{1}^{q+1} H_{k-1}+1\right\} \\
& \leq C\left\{\left[\int_{\Sigma_{t}}\left|\nabla\left(H_{1}^{\frac{q}{2}}\right)\right|^{2}+\int_{\Sigma_{t}} H_{1}^{q}\right]^{\frac{n-1}{n-3}}+\int_{\Sigma_{t}} H_{1}^{q+1} H_{k-1}+1\right\} .
\end{aligned}
$$

Therefore

$$
\begin{aligned}
& \left(\int_{M_{t}} H_{1}^{\frac{q(q+1)(n-1)}{(q+k)(n-3)}} H_{k-1}\right)^{\frac{n-3}{n-1}} \\
& \leq C\left\{\int_{\Sigma_{t}}\left|\nabla\left(H_{1}^{\frac{q}{2}}\right)\right|^{2}+\int_{\Sigma_{t}} H_{1}^{q+1} H_{k-1}+1\right\}
\end{aligned}
$$

for all $t \in I \cap J$.

Next, integrating with respect to $t$ over $I \cap J$ and using the coarea formula 
we get

$$
\begin{aligned}
& \int_{I \cap J}\left(\int_{M_{t}} H_{1}^{\frac{q(q+1)(n-1)}{(q+k)(n-3)}} H_{k-1}\right)^{\frac{n-3}{n-1}} d t \\
& \leq C\left\{\int_{M_{\rho}}\left|\nabla\left(H_{1}^{\frac{q}{2}}\right)\right|^{2}+\int_{M_{\rho}} H_{1}^{q+1} H_{k-1}+1\right\} \\
& \leq C \int_{M_{2 \rho}} H_{1}^{q+1} H_{k-1},
\end{aligned}
$$

where we have used (2.38) to estimate the gradient term, and where we have used that $H_{1}$ and $H_{k-1}$ are bounded away from zero.

We now estimate the left hand side from below in an obvious way, using the facts that $I \cap J \subset[\rho / 2, \rho]$ and $|I \cap J| \geq 3 \rho / 8$. After replacing $\rho / 2$ by $\rho$, we finally arrive at the iteration inequality

$$
\left(\int_{M_{\rho}} H_{1}^{\frac{q(q+1)(n-1)}{(q+k)(n-3)}} H_{k-1}\right)^{\frac{n-3}{n-1}} \leq C \int_{M_{4 \rho}} H_{1}^{q+1} H_{k-1}
$$

for all sufficiently small $\rho>0$. A straightforward calculation now shows that the exponent of $H_{1}$ on the left is greater than the exponent of $H_{1}$ on the right, provided $q+k>k(n-1) / 2((4.12)$ is satisfied with this choice). Moreover, the improvement in the exponent increases as $q$ increases. Therefore we may iterate (4.13) finitely many times to obtain a bound for the $L^{p}$ norm of $H_{1}$ on $M_{r}$ for small enough $r>0$, for some $p>k n / 2$. A bound for $H_{1}(0)$ then follows by appealing to the curvature bound of Section 2 .

In the case $k=2$ an almost identical argument leads to the estimate (4.13), because $H_{1}^{q+1} H_{k-1}=H_{1}^{q+2}=$ if $k=2$.

We now indicate the minor modifications that need to be made in the case $n=3$. In this case, by (4.1) with $\Sigma=\Sigma_{t}, w=H_{1}^{q / 2}$, and by Hölder's inequality we have, for any $r \in[0,2)$,

$$
\begin{aligned}
& \left(\int_{\Sigma_{t}} H_{1}^{\frac{q r}{2-r}}\right)^{\frac{2-r}{r}} \\
& \leq C(r)\left\{\int_{\Sigma_{t}}\left|\nabla_{E}^{\Sigma_{t}}\left(H_{1}^{\frac{q}{2}}\right)\right|^{r} d \sigma_{E}+\int_{\Sigma_{t}}\left|\mathbf{H}_{\Sigma_{t}}^{E}\right|^{r} H_{1}^{\frac{q r}{2}} d \sigma_{E}\right\}^{\frac{2}{r}} \\
& \leq C(r, \theta)\left\{\int_{\Sigma_{t}}\left|\nabla_{L}^{\Sigma_{t}}\left(H_{1}^{\frac{q}{2}}\right)\right|^{r} d \sigma_{L}+\int_{\Sigma_{t}}\left|\mathbf{H}_{\Sigma_{t}}^{E}\right|^{r} H_{1}^{\frac{q r}{2}} d \sigma_{L}\right\}^{\frac{2}{r}} \\
& \leq C(r, \theta)\left(\sigma_{L}\left(\Sigma_{t}\right)\right)^{\frac{2}{r}-1}\left\{\int_{\Sigma_{t}}\left|\nabla_{L}^{\Sigma_{t}}\left(H_{1}^{\frac{q}{2}}\right)\right|^{2} d \sigma_{L}+\int_{\Sigma_{t}}\left|\mathbf{H}_{\Sigma_{t}}^{E}\right|^{2} H_{1}^{q}\right\} d \sigma_{L} \\
& \leq C(r, \theta)\left(\frac{2 C_{1} \rho}{\epsilon}\right)^{\frac{2}{r}-1}\left\{\int_{\Sigma_{t}}\left|\nabla\left(H_{1}^{\frac{q}{2}}\right)\right|^{2} d \sigma_{L}+\rho^{-2} \int_{\Sigma_{t}} H_{1}^{q} d \sigma_{L}+\int_{\Sigma_{t}} H_{1}^{q+2} d \sigma_{L}\right\}
\end{aligned}
$$

for all $t \in I(\epsilon) \subset[\rho / 2, \rho]$, where we have used (4.9). The positive power of $\rho$ in the coefficient causes no difficulties in the subsequent argument. We now 
proceed exactly as before, with $(n-1) /(n-3)$ replaced by $r /(2-r)$ for any $r \in[1,2)$ such that

$$
\frac{q(q+1) r}{(q+k)(2-r)}>1
$$

this is automatically satisfied for any $q>0$, provided $r$ is sufficiently close to 2 . We arrive at the inequality

$$
\left(\int_{M_{\rho}} H_{1}^{\frac{q(q+1) r}{(q+k)(2-r)}} H_{k-1}\right)^{\frac{2-r}{r}} \leq C \int_{M_{4 \rho}} H_{1}^{q+1} H_{k-1}
$$

for all $r \in[1,2)$ sufficiently close to 2 , where now $C$ depends on $r$ in addition to the other quantities. There clearly is no need to iterate the inequality in this case. Notice, however, that $C \rightarrow \infty$ and $\rho \rightarrow 0$ as $r \rightarrow 2$.

This completes the proof of Theorem 1.1.

\section{References.}

[1] W. K. Allard, On the first variation of a varifold, Ann. Math. 95 (1972), 417-491.

[2] R. Bartnik, Existence of maximal surfaces in asymptotically flat spacetimes, Comm. Math. Phys. 94 (1984), 155-175.

[3] R. Bartnik, L. Simon, Spacelike hypersurfaces with prescribed boundary values and mean curvature, Comm. Math. Phys. 87 (1982), 131-152.

[4] P. Bayard, Problème de Dirichlet pour la courbure scalaire dans $\mathbf{R}^{3,1}$, C.R. Acad. Sci. Paris Sér. I Math. 332 (2001), 219-222.

[5] P. Bayard, Dirichlet problem for spacelike hypersurfaces with prescribed scalar curvature in $\mathbf{R}^{n, 1}$, Calc. Var. Partial Differ. Eqns (2003).

[6] S.-Y. Cheng, S.-T. Yau, Maximal spacelike hypersurfaces in the LorentzMinkowski spaces, Ann. Math. 104 (1976), 407-419.

[7] Ph. Delanoë, The Dirichlet problem for an equation of given LorentzGaussian curvature (Russian), Ukrain. Mat. Zh. 42 (1990), 1704-1710. Eng. transl.: Ukrain. Math. J. 42 (1991), 1538-1545.

[8] K. Ecker, G. Huisken, Parabolic methods for the construction of spacelike slices of prescribed mean curvature in cosmological spacetimes, Comm. Math. Phys. 135 (1991), 595-613. 
[9] H. Federer, Geometric measure theory, Springer, Berlin-Heidelberg, 1969.

[10] C. Gerhardt, $H$-surfaces in Lorentzian manifolds, Comm. Math. Phys. 89 (1983), 523-553.

[11] C. Gerhardt, Hypersurfaces of prescribed mean curvature in Lorentzian manifolds, Math. Z. 235 (2000), 83-97.

[12] C. Gerhardt, Hypersurfaces of prescribed curvature in Lorentzian manifolds, Indiana Univ. Math. J. 49 (2000), 1125-1153.

[13] B. Guan, The Dirichlet problem for Monge-Ampère equations in nonconvex domains and spacelike hypersurfaces of constant Gauss curvature, Trans. Amer. Math. Soc. 350, (1998), 4955-4971.

[14] J. H. Michael, L. M. Simon, Sobolev and mean value inequalities on generalized submanifolds of $\mathbf{R}^{n}$, Comm. Pure Appl. Math. 26 (1973), $361-379$.

[15] D. S. Mitrinović, Analytic inequalities, Springer, Berlin, 1970.

[16] L. Simon, Lectures on geometric measure theory, Proceedings of the Centre for Mathematical Analysis, Australian National University, 3 (1983).

[17] O. C. Schnürer, The Dirichlet problem for Weingarten hypersurfaces in Lorentz manifolds, Math. Z. 242 (2002), 159-181.

[18] J. Urbas, Regularity of generalized solutions of Monge-Ampère equations, Math. Z. 197 (1988), 365-393.

[19] J. Urbas, An interior curvature bound for hypersurfaces of prescribed $k$-th mean curvature, J. Reine Angew. Math. 519 (2000), 41-57.

[20] J. Urbas, Monotonicity formulae and curvature equations, J. Reine Angew. Math. 557 (2003), 199-218.

Centre for Mathematics and its Applications

School of Mathematical Sciences

Australian National University

Canberra ACT 0200

Australia

E-mail: John.Urbas@maths.anu.edu.au 